\title{
Interface resistances of magnetic multilayers
}

\author{
Kees M. Schep* \\ Faculty of Applied Physics and Delft Institute of Microelectronics and Submicrontechnology, Delft University of Technology, \\ Lorentzweg 1, 2628 CJ Delft, The Netherlands \\ Jeroen B. A. N. van Hoof \\ Department of Theoretical Physics, University of Nijmegen, Toernooiveld, 6525 ED Nijmegen, The Netherlands \\ Paul J. Kelly \\ Philips Research Laboratories, Prof. Holstlaan 4, 5656 AA Eindhoven, The Netherlands \\ Gerrit E. W. Bauer \\ Faculty of Applied Physics and Delft Institute of Microelectronics and Submicrontechnology, Delft University of Technology, \\ Lorentzweg 1, 2628 CJ Delft, The Netherlands \\ John E. Inglesfield \\ Department of Physics and Astronomy, University of Wales, P.O. Box 913, Cardiff CF2 3YB, United Kingdom
}

(Received 12 June 1997)

\begin{abstract}
Perpendicular transport in disordered magnetic multilayers is studied by combining first-principles electronic-structure calculations with the Boltzmann equation. Resistor-model-type expressions for the multilayer resistance are derived and interface resistances are calculated without using any adjustable parameters. Experimentally observed interface resistances can be explained largely in terms of specular interface scattering and diffuse bulk scattering. [S0163-1829(97)06337-6]
\end{abstract}

The resistance of magnetic metallic multilayers drops sharply when the alignment of adjacent ferromagnetic layers is changed from antiparallel to parallel by applying an external magnetic field. ${ }^{1,2}$ The study of this so-called giant magnetoresistance (GMR) effect is driven by the interest of industry in improved magnetoresistive sensors, as well as by scientific curiosity.

Whereas spin-valve structures are most promising for applications, ${ }^{3}$ multilayers in which the current flows perpendicular to the interfaces are preferred for fundamental studies. ${ }^{4-10}$ This current-perpendicular-to-plane (CPP) geometry has the advantage over the more conventional currentin-plane (CIP) geometry that the interpretation of the experimental measurements is simpler. The way in which the CPP resistance varies as layer thicknesses are changed can be fitted quite well by a simple resistor model. For a nonmagnetic multilayer composed of materials $\mathrm{A}$ and $\mathrm{B}$ with resistivities $\rho_{\mathrm{A}}$ and $\rho_{\mathrm{B}}$ and thicknesses $d_{\mathrm{A}}$ and $d_{\mathrm{B}}$, respectively, the resistor model for the total multilayer resistance $R_{T}$ times the cross sectional area $A$ reads ${ }^{11}$

$$
A R_{T}=M\left[\rho_{\mathrm{A}} d_{\mathrm{A}}+A R_{\mathrm{A} / \mathrm{B}}+\rho_{\mathrm{B}} d_{\mathrm{B}}+A R_{\mathrm{A} / \mathrm{B}}\right],
$$

in which $M$ is the number of bilayers and $R_{\mathrm{A} / \mathrm{B}}$ is the resistance of an A/B interface. The total resistance is thus simply a sum of bulk and interface resistances. If some of the layers are magnetic, the resistor model should be extended to include spin polarization. ${ }^{6}$ By measuring the total resistance as a function of the layer thicknesses, the bulk resistivities and interface resistances can be determined experimentally. The interface resistance turns out to be strongly spin dependent and it dominates the resistance and magnetoresistance for layer thicknesses which are not too large. ${ }^{8,9}$ Understanding CPP magnetoresistance is then largely a matter of understanding the origin of the interface resistance.

In spite of the usefulness of the resistor model for analyzing experimental data, the microscopic origin of the parameters it contains remains unclear. The choice of a model for the disorder to be used in a detailed theoretical description is made nontrivial by a lack of experimental information; it has turned out to be very difficult to characterize the interfaces in terms of different types of disorder such as intermixing, short-range geometrical roughness and terrace formation.

There are several microscopic mechanisms that can give rise to the interface resistance. The most frequently invoked mechanism is diffuse scattering at interdiffused atoms or interfacial roughness. Free electron theories based on this type of scattering yield the resistor model of Eq. (1) as well as its spin polarized extension, ${ }^{1-14}$ thus providing a good fit to the experimental data. Unfortunately, the value of such a fit is limited because the free electron theories omit an important feature of transition metals, namely, their complex electronic structures. There have been a number of attempts to take account of this complexity which, though they differ in the way they account for disorder, all lead to the conclusion that the spin dependence of the electronic structure makes a large contribution to the magnetoresistance. ${ }^{15-20}$ However, no attempt has been made to explain the experimentally observed layer thickness dependences, even qualitatively, using these approaches. Consequently, beyond establishing the importance of band structure effects in the study of GMR, their relevance to the above-mentioned experiments is not clear. It is the purpose of this paper to present a framework for cal- 
culating multilayer resistances within which (i) the full electronic structure is taken into account from first principles, (ii) an expression of the form Eq. (1) is derived and (iii) parameter-free estimates of the interface resistances are made.

In real multilayer structures the mean-free number of traversed interfaces is not much larger than the number of interfaces in a multilayer period. ${ }^{21}$ This implies that the electrons scattered from consecutive interfaces do not interfere coherently to form multilayer Bloch states and the picture of a multilayer band structure should be abandoned. ${ }^{22}$ In contrast to previous $a b$ initio methods we will therefore take the electronic structure into account without imposing periodicity in the growth direction. To avoid introducing arbitrary fitting parameters we calculate the scattering at a single interface from first principles. Because the microscopic details of the disorder which gives rise to scattering in the bulk of the layers are not known, a simple model from random matrix theory is used to describe diffusive transport through the bulk. The propagation between adjacent interfaces will be treated semiclassically by using the scattering properties of a single interface as boundary conditions for the Boltzmann equation. ${ }^{23}$

We calculate the current in response to a density gradient, taking the drift term due to the force in the Boltzmann equation equal to zero. For a multilayer grown in the $z$ direction the distribution function does not depend on the $x$ and $y$ coordinates because of the translational invariance along the interface planes. In the plane $L$ at $z=z_{L}$ the distribution function is denoted by $f_{L, i}^{ \pm}$where the + and - signs label right and left going states which move in positive and negative $z$ directions, respectively. The index of the electron state $i$ is a shorthand notation for the component of the bulk Bloch vector parallel to the interface, $\vec{k}_{\|}$, and the index $\nu$ that labels different sheets of constant energy surface. All electron states are normalized to carry unit flux in the direction perpendicular to the interfaces. For low current densities the deviation of $f_{L, i}^{ \pm}$from its equilibrium value $f_{L, i}^{0}$ is small and restricted to a small region near the Fermi energy $E_{F}$. The distribution function can be written as

$$
f_{L, i}^{ \pm}=f_{L, i}^{0}+\delta\left(\varepsilon_{L, i}-E_{F}\right)\left[\mu_{L}-\mu^{0}+g_{L, i}^{ \pm}\right],
$$

with $\varepsilon_{L, i}$ the energy of state $i$ in plane $L$. The deviation of the distribution function from equilibrium is divided into an isotropic chemical potential difference $\mu_{L}-\mu^{0}$ and an anisotropic term $g_{L, i}^{ \pm}$.

To calculate the resistance of an infinite $\mathrm{A} / \mathrm{B}$ multilayer we consider the single multilayer period depicted schematically in Fig. 1. We include the diffuse bulk scattering and the interface scattering in the same way, namely via boundary conditions for the distribution function. In this way we only need to calculate $f_{L, i}^{ \pm}$in a finite number of planes and not as a continuous function of $z$ which is a major simplification. The distribution functions in planes $L$ and $L^{\prime}$ are connected via the boundary conditions

$$
f_{L^{\prime}, i}^{+}=\sum_{j \in L}\left(T_{L L^{\prime}}\right)_{i j} f_{L, j}^{+}+\sum_{j \in L^{\prime}}\left(R_{L L^{\prime}}^{\prime}\right)_{i j} f_{L^{\prime}, j}^{-},
$$

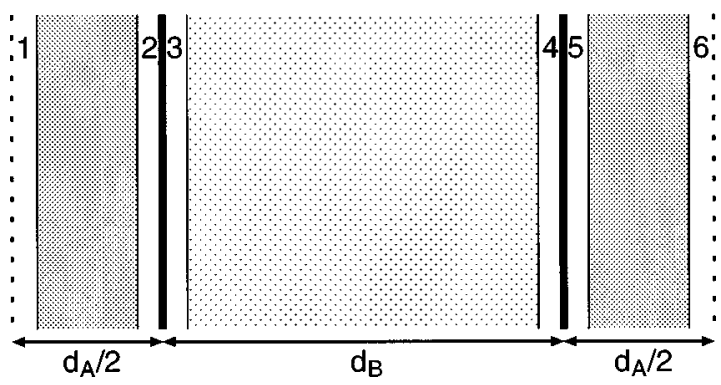

FIG. 1. Schematic representation of one multilayer period of an infinite $\mathrm{A} / \mathrm{B}$ multilayer with diffuse bulk scattering. The thick lines represent the interfaces. The dotted areas represent the bulk regions with diffuse scattering. Periodic boundary conditions are applied at the dashed lines. The numbered planes are used in the text for the derivation of Eq. (6).

$$
f_{L, i}^{-}=\sum_{j \in L}\left(R_{L L^{\prime}}\right)_{i j} f_{L, j}^{+}+\sum_{j \in L^{\prime}}\left(T_{L L^{\prime}}^{\prime}\right)_{i j} f_{L^{\prime}, j}^{-} .
$$

The elements $i j$ of the matrices $T_{L L^{\prime}}$ and $R_{L L^{\prime}}\left(T_{L L^{\prime}}^{\prime}\right.$ and $R_{L L^{\prime}}^{\prime}$ ) contain the transmission and reflection probabilities of the incoming state $j$ from the left (right) to an outgoing state $i$. By combining the boundary conditions between planes $L$ and $L^{\prime}$ with those between planes $L^{\prime}$ and $L^{\prime \prime}$ effective boundary conditions can be derived that connect the distribution functions in planes $L$ and $L^{\prime \prime}$. This combination of boundary conditions is equivalent to the semiclassical concatenation of scattering matrices in the Landauer-Büttiker formalism. ${ }^{24}$

The precise form of the boundary conditions for the diffusive bulk scattering depends on the scattering mechanism. Because we know very little about the disorder we follow an approach motivated by random matrix theory ${ }^{25}$ in which a complete and isotropic mixing of states with different index $i$ is assumed. The diffusive bulk regions are characterized by

$$
\begin{aligned}
& T_{i j}=T_{i j}^{\prime}=\frac{1}{N} \frac{1}{1+s}, \\
& R_{i j}=R_{i j}^{\prime}=\frac{1}{N} \frac{s}{1+s},
\end{aligned}
$$

in which $s=\rho d N e^{2} / A h$ with $N$ the number of conduction channels. This model should be constrasted with the case of ballistic bulk propagtion in which $T_{i j}=\delta_{i j}$ and $R_{i j}=0$. Equations (4) incorporate the most important feature of diffuse scattering: the mixing of states with different $\vec{k}$. In most previous $a b$ initio calculations the scattering in the bulk was not evaluated for a specific microscopic mechanism but treated phenomenologically using state independent relaxation times, ${ }^{15,19}$ mean free paths, ${ }^{17}$ or self-energies. ${ }^{20}$ The parameter $s$ which characterizes the strength of the diffuse scattering in Eqs. (4) will not appear in the final results for the interface resistance so our approach remains parameter free.

For an infinite A/B multilayer the periodic boundary conditions $g_{6, i}^{ \pm}=g_{1, i}^{ \pm}$hold over one multilayer period as shown in Fig. 1. By combining this with the effective boundary conditions (3) connecting $f_{1, i}^{ \pm}$and $f_{6, i}^{ \pm}$a solution for the distribu- 
TABLE I. Interface resistances (in units of $\mathrm{f} \Omega \mathrm{m}^{2}$ ) of (100) and (111) oriented $\mathrm{Co} / \mathrm{Cu}$ multilayers as calculated for diffuse and ballistic bulk propagation, compared with the experimental values obtained from Ref. 8.

\begin{tabular}{lccc}
\hline \hline System & Orientation & Majority & Minority \\
\hline Calculated diffuse & $(100)$ & 0.35 & 1.90 \\
Calculated diffuse & $(111)$ & 0.40 & 1.35 \\
Calculated ballistic & $(100)$ & 0.0005 & 0.54 \\
Calculated ballistic & $(111)$ & 0.0001 & 0.64 \\
Experimental & $(111)$ & $0.26 \pm 0.06$ & $1.84 \pm 0.14$ \\
\hline \hline
\end{tabular}

tion function is obtained in terms of $\mu_{1}-\mu_{6}$. The current density $J$ can be calculated from

$$
J=\frac{e}{h A} \sum_{i}\left[g_{L, i}^{+}-g_{L, i}^{-}\right]
$$

The ratio of the drop in chemical potential and the current density yields the resistance per bilayer: $A R_{T} / M=\left(\mu_{1}\right.$ $\left.-\mu_{6}\right) / e J$. From a straightforward calculation we find that the total resistance of the multilayer can be written as Eq. (1) with the interface resistance given by

$$
A R_{\mathrm{A} / \mathrm{B}}=\frac{A h}{e^{2}}\left[\frac{1}{\sum_{i j}\left(T_{23}\right)_{i j}}-\frac{1}{2}\left(\frac{1}{N_{\mathrm{A}}}+\frac{1}{N_{\mathrm{B}}}\right)\right] .
$$

The resistor model is retrieved due to the assumptions of lack of phase coherence and of completely diffuse scattering in the bulk of the layers. Equation (6) expresses the interface resistance in terms of the single interface transmission probabilities. This expression does not contain any bulk parameters and thus provides a parameter-free estimate for the resistance of an interface embedded in a diffusive scattering environment modelled by Eqs. (4). Similar expressions were derived in Ref. 26 but only for a specular barrier in a free electron description in the limiting cases of either weak or strong reflection. In contrast Eq. (6) is valid for both specular and diffuse interface scattering of arbitrary strength and for general band structures which need not be identical on both sides of the interface. This universal applicability of Eq. (6) is what makes it suitable for combination with the results obtained from $a b$ initio calculations.

In order to arrive at concrete results the transmission and reflection probabilities have to be calculated. Our present calculations are restricted to ideal interfaces without any defects for which the scattering is specular, i.e., all the scattering matrices are diagonal in $\vec{k}_{\|}$. The electronic structure of a single interface is calculated in the local-spin-density approximation using a surface-embedding Green's function technique. ${ }^{27}$ From the Green's function the scattering properties of the interface are extracted. A detailed description of this formalism will be given elsewhere. ${ }^{28}$ Alternative ways of calculating reflection and transmission at a single specular interface have been used recently by Bruno ${ }^{29}$ and Stiles. ${ }^{30}$

Evaluation of Eq. (6) for (100) and (111) oriented $\mathrm{Co} / \mathrm{Cu}$ multilayers yields interface resistances as summarized in Table I together with the values obtained from experiment. The lower values for the majority spin compared to the minority spin is a result of the better matching of the $\mathrm{Co}$ and $\mathrm{Cu}$ bulk bands for this spin direction which results in higher transmission probabilities. The calculations for the (111) orientation are in good agreement with the experimental results. We emphasize that this agreement is obtained without invoking any fit parameters. The calculated difference in the minority spin interface resistance between the (100) and the (111) orientation should be measurable.

While most previous theories have focused on the effect of short-length-scale interface roughness, the contribution to the interface resistance of an ideal interface has been studied previously using free electron calculations which included a potential step at the interface. ${ }^{31,32}$ These model calculations are very useful to identify the possible microscopic mechanisms that can give rise to interface resistance. For making quantitative comparisons they are, however, of limited use because they contain adjustable parameters such as the heights of the spin-dependent potential steps and effective masses of the different materials. In contrast our calculation of the interface resistances does not contain any free parameters. Since interface roughness is completely omitted in the present theory we may conclude from our numerical results that to obtain agreement with experiment for interface resistances, it is not necessary; small interface transmittivity can be largely explained by the specular reflection from the "potential steps" at the interfaces. We cannot conclude, however, that interface roughness is negligible, since it may be instrumental in achieving the diffusivity which we here attribute to the bulk material.

We can also calculate the multilayer resistance for the situation that the bulk propagation is ballistic. The dephasing of the electron wave function can in this case be due to, for example, inelastic scattering. Random variations of the layer thicknesses that arise from, for instance, terrace formation and break the translational invariance of the multilayer also contribute to the averaging over the phase an electron acquires when traversing the bulk. ${ }^{33} \mathrm{~A}$ straightforward calculation similar to the one for the diffuse bulk transmission yields an expression for the interface resistance which is a generalization of the result obtained already by Landauer for a single symmetric planar barrier in a free electron description. ${ }^{34}$ There are no bulk contributions to the resistance because of the ballistic transmission. In this regime the resistance originates exclusively from the specular reflection at the interfaces. The calculated interface resistances for (100) and (111) oriented $\mathrm{Co} / \mathrm{Cu}$ multilayers are summarized in Table I. The comparison with experiment reveals that the microscopic mechanism of specular interface scattering in combination with purely ballistic bulk transmission cannot account for the experimental values. Some amount of diffuse scattering should be taken into account. 
The values for the interface resistance are different for the situations with and without diffuse bulk scattering which implies that the interface resistance can be different for thick and thin bulk layers. Although this fact is known in the literature (e.g., in Ref. 35) it has not received much attention in the field of magnetic multilayers. ${ }^{36}$ This size effect may explain the deviations from the resistor model observed in some experiments. ${ }^{7,9}$

Recently, Mathon studied perpendicular transport in multilayers with random layer thicknesses ${ }^{37}$ and realistic band structures. In contrast to the present paper he assumed complete quantum coherence which, combined with specular interfaces, leads to Anderson localization of the wave functions. Which approach is relevant for real multilayers can easily be determined experimentally by growing multilayers intentionally with random layer thicknesses. Our approach predicts that this additional randomness does not change the parameters of the resistor model, whereas Mathon predicts a large increase of the resistance and the magnetoresistance. ${ }^{37}$

In summary, based on a semiclassical theory of perpendicular transport parameter-free estimates are obtained for the interface resistances. For $\mathrm{Co} / \mathrm{Cu}$ the experimentally observed values are in good agreement with the microscopic mechanism of specular interface scattering in combination with diffuse bulk scattering.

This work was part of the research program of the "Stichting voor Fundamenteel Onderzoek der Materie (FOM),", which was financially supported by the "Nederlandse Organisatie voor Wetenschappelijk Onderzoek (NWO)." We acknowledge benefits from the TMR Research Network on \{lit Interface Magnetism\} under Contract No. FMRX-CT960089 (DG12-MIHT).
*Also at: Philips Research Laboratories, Prof. Holstlaan 4, 5656 AA Eindhoven, The Netherlands.

${ }^{1}$ M. N. Baibich et al., Phys. Rev. Lett. 61, 2472 (1988); G. Binasch et al., Phys. Rev. B 39, 4828 (1989).

${ }^{2}$ P. M. Levy, Solid State Phys. 47, 367 (1994).

${ }^{3}$ B. Dieny, J. Magn. Magn. Mater. 136, 335 (1994).

${ }^{4}$ W. P. Pratt Jr. et al., Phys. Rev. Lett. 66, 3060 (1991).

${ }^{5}$ M. A. M. Gijs, S. K. J. Lenczowski, and J. B. Giesbers, Phys. Rev. Lett. 70, 3343 (1993).

${ }^{6}$ S.-F. Lee et al., J. Magn. Magn. Mater. 118, L1 (1993).

${ }^{7}$ P. A. Schroeder et al., in Magnetic Ultrathin Films, Multilayers, and Surfaces/Interfaces and Characterization, edited by B. T. Jonker et al., Symposia Proceedings No. 313 (Materials Research Society, Pittsburgh, 1993), p. 47.

${ }^{8}$ Q. Yang et al., Phys. Rev. B 51, 3226 (1995).

${ }^{9}$ S.-F. Lee et al., Phys. Rev. B 52, 15426 (1995).

${ }^{10}$ For a recent review see M. A. M. Gijs and G. E. W. Bauer, Adv. Phys. 46, 285 (1997).

${ }^{11}$ S. Zhang and P. M. Levy, J. Appl. Phys. 69, 4786 (1991).

${ }^{12}$ G. E. W. Bauer, Phys. Rev. Lett. 69, 1676 (1992).

${ }^{13}$ H. E. Camblong, S. Zhang, and P. M. Levy, Phys. Rev. B 47, 4735 (1993).

${ }^{14}$ T. Valet and A. Fert, Phys. Rev. B 48, 7099 (1993).

${ }^{15}$ T. Oguchi, J. Magn. Magn. Mater. 126, 519 (1993).

${ }^{16}$ W. H. Butler, J. M. MacLaren, and X.-G. Zhang, in Magnetic Ultrathin Films, Multilayers, and Surfaces/Interfaces and Characterization (Ref. 7), p. 59.

${ }^{17}$ R. K. Nesbet, J. Phys. Condens. Matter 6, L449 (1994).

${ }^{18}$ K. M. Schep, P. J. Kelly, and G. E. W. Bauer, Phys. Rev. Lett. 74, 586 (1995).

${ }^{19}$ P. Zahn et al., Phys. Rev. Lett. 75, 2996 (1995).
${ }^{20}$ W. H. Butler et al., Phys. Rev. Lett. 76, 3216 (1996)

${ }^{21}$ G. E. W. Bauer, K. M. Schep, and P. J. Kelly, J. Magn. Magn. Mater. 151, 369 (1995).

${ }^{22}$ P. M. Levy and S. Zhang, J. Magn. Magn. Mater. 151, 315 (1995).

${ }^{23}$ This procedure is analogous to the calculation of the residual resistivities in dilute alloys in which the (elastic) scattering properties of a single impurity are calculated quantum mechanically and then combined with the Boltzmann equation to account for the random distances between the impurities; see e.g., I. Mertig, R. Zeller, and P. H. Dederichs, Phys. Rev. B 47, 16178 (1993).

${ }^{24}$ M. Cahay, M. McLennan, and S. Datta, Phys. Rev. B 37, 10125 (1988).

${ }^{25}$ P. A. Mello and A. D. Stone, Phys. Rev. B 44, 3559 (1991).

${ }^{26}$ B. Laikhtman and S. Luryi, Phys. Rev. B 49, 17177 (1994).

${ }^{27}$ S. Crampin, J. B. A. N. van Hoof, M. Nekovee, and J. E. Inglesfield, J. Phys. Condens. Matter 4, 1475 (1992).

${ }^{28}$ J. B. A. N. van Hoof et al. (unpublished).

${ }^{29}$ P. Bruno, Phys. Rev. B 52, 411 (1995).

${ }^{30}$ M. D. Stiles, J. Appl. Phys. 79, 5805 (1996).

${ }^{31}$ J. Barnaś and A. Fert, Phys. Rev. B 49, 12835 (1994).

${ }^{32}$ V. K. Dugaev, V. I. Litvinov, and P. P. Petrov, Phys. Rev. B 52, 5306 (1995).

${ }^{33}$ This type of phase averaging was also invoked in calculations of the interlayer exchange coupling; see e.g., P. Lang et al., Phys. Rev. B 53, 9092 (1996).

${ }^{34}$ R. Landauer, IBM J. Res. Dev. 1, 223 (1957).

${ }^{35}$ R. Landauer, Phys. Rev. B 52, 11225 (1995).

${ }^{36}$ K. M. Schep and G. E. W. Bauer, Bull. Am. Phys. Soc. 41, 131 (1996); (unpublished).

${ }^{37}$ J. Mathon, Phys. Rev. B 55, 960 (1997). 